\title{
Relations publiques: stratégies de rupture et construction de l'objet
}

Yves Winkin

\section{(2) OpenEdition}

1 Journals

Édition électronique

URL : http://journals.openedition.org/communicationorganisation/1655

DOI : 10.4000/communicationorganisation. 1655

ISSN : $1775-3546$

Éditeur

Presses universitaires de Bordeaux

Édition imprimée

Date de publication : 1 novembre 1993

ISSN : 1168-5549

Référence électronique

Yves Winkin, "Relations publiques: stratégies de rupture et construction de l'objet », Communication et organisation [En ligne], 4 | 1993, mis en ligne le 26 mars 2012, consulté le 02 mai 2019. URL : http:// journals.openedition.org/communicationorganisation/1655; DOI : 10.4000/ communicationorganisation. 1655

Ce document a été généré automatiquement le 2 mai 2019.

(c) Presses universitaires de Bordeaux 


\title{
Relations publiques: stratégies de rupture et construction de l'objet
}

\author{
Yves Winkin
}

1 Sans doute l'ambiance "positiviste » dans laquelle baigne le Métier de sociologue de Pierre Bourdieu, Jean-Claude Chamboredon et Jean-Claude Passeron paraît bien surannée aujourd'hui ${ }^{1}$. Déjà lointaine semble l'époque où les sociologues tenaient le haut du pavé et s'exprimaient volontiers en termes de "rupture avec le sens commun $»^{2}$. Mais il ne faut pas jeter le bébé avec l'eau du bain: le message que les trois sociologues voulaient transmettre reste essentiel aujourd'hui; il prolonge ses racines dans la tradition épistémologique de Durkheim, Fauconnet et Mauss d'une part, de Duhem, Bachelard et Canguilhem, d'autre part ${ }^{3}$. Poser que toute démarche scientifique est fondée sur une lutte contre «l'illusion du savoir immédiat» n'a rien d'arrogant, même en ces temps d'ethnométhodologie plus ou moins bien comprise. Dire que la science «construit» ses objets ne procède pas d'un discours impérialiste, même si l'heure est au récit spontané, à la «théorie fondée » (grounded theory) et à l'essai d'humeur.

2 Les principes énoncés par Bourdieu, Chamboredon et Passeron restent tout particulièrement pertinents dans le champ des sciences de l'information et de la communication, qui est encore trop souvent traversé par des confusions entre problèmes sociaux et problématiques théoriques, entre démarche de recherche et démarche d'intervention, entre savoir et savoir-faire, cette dernière confusion est particulièrement patente dans le secteur des relations publiques, qui sont enseignées dans les universités comme dans les écoles privées, dans les départements de communication comme dans les sections de marketing. Il suffit de parcourir la littérature qui s'y enseigne pour être édifié : c'est le règne des " cas célèbres ", des anecdotes et des prescriptions plus ou moins implicites. Certes, on perçoit une volonté de «théorisation", mais les emprunts sont souvent empruntés, gauches et maladroits (un schéma de Shannon par-ci, un « two-step flow » par là...). Il me paraît qu'il faut réagir, non parce que ce savoir demi-savant se vend bien (sous la forme de cours, de consultances, de séminaires, etc.) mais parce qu'il nuit au développement de la recherche en sciences de l'information et de la communication pourvu que l'on considère que les relations publiques (et plus globalement la 
communication dans et par les organisations) ressortissent à ce champ du savoir en voie de constitution. Ma réaction passera non par une critique de la littérature existante ${ }^{4}$, mais par la présentation d'un ensemble de stratégies de rupture, restant en cela très fidèle à la vision du Métier de sociologue et des textes qui y sont associés.

\section{Stratégie de rupture}

3 Face à la présentation enchantée des relations publiques qu'offre le discours professionnel, la première stratégie de rupture est celle de l'analyse historique: où, quand et comment les relations publiques ont-elles émergé, en tant qu'ensemble relativement autonome de discours et de pratiques? La réponse à cette question semble fournie dans le premier chapitre de tous les manuels de relations publiques. Mais le lecteur critique s'aperçoit vite que les auteurs, issus du sérail, ne présentent que leur vision des faits ou ne font que reprendre la présentation historique de X, qui lui-même, etc. Très rares encore sont les travaux de recherche sur l'émergence des relations publiques aux États-Unis et en Europe. Il n'est pas question de suggérer ici la rédaction d'un gros ouvrage de synthèse : à ce stade, il faut plutôt encourager la production de chapitres de cette histoire de relations publiques, notamment sous la forme de mémoires et de thèses ${ }^{5}$. Ces travaux peuvent emprunter de multiples voies, aussi bien celles de l'histoire orale (analyse d'entretiens approfondis avec des "pères fondateurs", par exemple) que celle de l'exploration des archives (d'une entreprise, d'un cabinet-conseil etc), aussi bien celles de la monographie (histoire des relations publiques dans un secteur précis, à une certaine époque, dans une région donnée, etc; histoire des relations publiques de telle entreprise, de telle institution publique, etc) que celle du panorama général nourri par une hypothèse organisatrice ${ }^{6}$. Ce qui est essentiel, c'est le principe d'un regard dédoublé : les relations historiques offertes par leurs acteurs sont souvent intéressantes quant aux matériaux semi-bruts qu'elles relèvent mais elles ne peuvent se substituer aux recherches menées par des tiers non pour des raisons d'« objectivité" (après tout, une subjectivité chasse l'autre, pour faire allusion à Weber et Aron) mais pour des raisons de distance à l'objet, comme en anthropologie, où le discours de l'informateur ne peut se substituer à celui de l'anthropologue, même si celui-ci laisse de plus en plus la parole à celui-là7.

4 La démarche historique peut susciter une deuxième stratégie de rupture en offrant aux chercheurs des modèles d'analyse qui, par glissement analogique, peuvent faire subir une cohérence d'un ensemble de faits épars. Ainsi, Axel Gryspeerdt a utilisé l'idée d'« insiders » "outsiders» que l'historien américain Simon Schama propose dans L'embarras des richesses: la culture hollandaise au Siècle d'Or (Paris, Gallimard, 1991) pour étudier «les relations entre in et out, internes et externes »:

«Les personnes représentées ou évoquées, dans la publicité institutionnelle et dans les toiles de maîtres hollandais (là où apparaissent les insiders' outsiders) témoignent en effet d'un même respect face aux éléments transcendants (le respect qui anime les citoyens pour leur ville est commun à chacun d'eux quel que soit le statut social), partagent de la déférence les uns vis-à-vis des autres et sont légitimés à la fois par une instance supérieure et par le regard porté sur elles par d'autres ou par elles-mêmes $»^{8}$.

Dans une veine similaire, j'ai emprunté la notion de « curialisation » à Norbert Elias pour poser la question : 
«Ne pourrait-on voir dans la professionnalisation actuelle des relations publiques un processus de curialisation adopté à notre époque? (...), De même que la curialisation a isolé les nobles de leur région d'origine et les a constitués en groupe distinct, on pourrait avancer que la professionnalisation des relations publiques a entraîné la production de manifestations par et pour des "professionnels de la représentation $»^{9}$.

6 Ces juxtapositions ne relèvent évidemment pas de l'histoire : les relations publiques ne sont pas à leurs visiteurs ce que les bourgeois hollandais étaient à leurs pauvres - et il n'y a pas de continuité historique entre les cours princières du XVIIe siècle et les cocktails de presse $\mathrm{du} \mathrm{XX}^{\mathrm{e}}$ siècle. Elles n'ont également aucune vertu explicative: elles sont simplement offertes pour «faire penser » - mais faire penser autrement. Il s'agit bien d'« analogies méthodologiques » au sens où De Coster l'entend : ce sont des instruments d'invention ${ }^{10}$. On pourrait ajouter : et de rupture. Ainsi, la notion d'« insiders' outsiders » appliquée à l'univers des relations publiques permet de rompre avec la perception commune et de reconfigurer différemment les rapports entre les acteurs présents. Il n'y a plus deux groupes en présence, mais un seul, uni dans un même respect de l'entreprise. Similairement penser les manifestations de relations publiques comme des phénomènes de cour permet de saisir une dimension de ces rassemblements formels : c'est qu'ils se produisent entre « professionnels de la représentation ». Ces « événements » rassemblent moins des amateurs et des professionnels que des professionnels entre eux. Si tous ne font pas de RP, beaucoup font profession d'être constamment en situation publique. À partir de cette proposition, volontiers iconoclaste (mais «toute sociologie est une socioloclastie ", a dit Bourdieu dans sa leçon inaugurale au Collège de France), il faut mener un vrai travail de terrain - qui attend toujours ses ethnographes.

7 La troisième stratégie de rupture est également faite de rapprochements iconoclastes. Même objectif : faire réfléchir autrement, désamorcer les réflexes de pensée, obliger à repartir d'ailleurs. Les relations publiques aiment se présenter comme " une profession ", au sens noble de la langue sociologique américaine d'obédience parsonienne. Il faut, à l'instar d'Everett Hugues qui voulait que ses étudiants établissent des rapprochements entre médecins et concierges, psychiatres et prostituées, afin de déjouer les discours d'auto-célébration, se demander pourquoi les relations publiques ne relèvent pas, par exemple, des métiers de la publicité, du secteur HORECA ou de l'économie domestique. La question paraît presque injurieuse : si elle ne fait pas rire, c'est qu'elle lève un coin de voile. Pourquoi les professionnels des relations publiques mettent-ils toujours autant de soin à opérer la distinction entre eux et leurs collègues « qui travaillent dans la pub»? Peut-être parce qu'ils en sont objectivement très proches. In fine, pour les uns et les autres, il s'agit de contribuer à l'augmentation du chiffre d'affaires de l'entreprise, quels que soient les moyens utilisés, directs ou détournés, agressifs ou euphorisants-Même dans le secteur non marchand (universités, hôpitaux ou asbl culturelles), la finalité reste économique: plus d'étudiants, de malades ou de membres, cela signifie crûment plus d'allocations, plus de rentrées, plus de crédits. La vérité des relations publiques y apparaît d'ailleurs de façon encore plus transparente : les relations publiques doivent se substituer à la publicité parce que l'éducation, la santé et, dans une moindre mesure, la culture, acceptent encore mal d'être « vendues comme des savonnettes ». Aux relations publiques, dès lors, de les vendre autrement, c'est-à-dire comme si elles s'offraient, dans le nimbe d'une société enfin débarrassée de ses médiocres rapports économiques, à ceux qui les désirent ou en ont besoin. 
Le rapprochement, au moins aussi scandaleux, avec le secteur HORECA (Hôtels Restaurants - Cafés) permettrait, au moins dans un premier temps, de mieux prendre en charge la dimension festive, ludique, sinon érotique, des pratiques de relations publiques. Tout se passe comme si les professionnels des relations publiques, conscients de l'inévitable réduction de leur travail, essentiellement souterrain et très ingrat, à un déploiement de petits fours et de mousseux, déniaient dans leur discours la réalité du plaisir que leurs actions peuvent susciter : plaisir des yeux, plaisir du ventre, plaisir des fantasmes qui s'éveillent... Le tout sans bourse délier : faut-il insister sur l'importance de la gratuité des opérations de relations publiques? On finit par oublier cet aspect des choses, alors qu'il est essentiel. Le rapprochement avec d'autres lieux semi-publics de restauration et de séduction, qui sont nécessairement payants, rappelle encore une fois que les relations publiques évoluent au sein d'une économie du don et du contre-don (nécessairement différé, comme l'a souvent rappelé P. Bourdieu), en apparente contradiction avec le système économique qui les a engendrées et développées.

Mais le parallélisme avec le secteur HORECA évacue une autre dimension importante des pratiques de relations publiques : la sélection des invités (du moins en apparence). C'est ici que le rapprochement avec l'économie domestique permet de mieux cerner la logique des relations publiques, tout particulièrement en ce qui concerne le contre-don.

On ne sort pas d'un restaurant sans payer - mais on y entre sans invitation (dans la plupart des cas). On sort d'une manifestation de relations publiques sans payer - mais on n'y entre pas sans invitation (en principe). Exactement comme dans une soirée privée : les invitations sont personnalisées et il serait du plus mauvais goût de faire payer ses hôtes à la fin du repas... On retrouve ici l'idée, souvent entretenue par le discours «relations publiques », de la " grande famille », qui s'illustre dans les comportements professionnels, par des accueils " personnalisés ", « chaleureux ", « engagés ", etc. Mais là ne s'arrête pas la comparaison avec l'économie domestique. Une soirée chez des amis fonctionne sans doute sur le modèle du don (le repas, les alcools, etc.), contre-don (les fleurs, la bouteille de vin ou la participation à la plonge), mais le cycle peut se clôturer dès la fin de la soirée. Par contre, une soirée dans la famille ou la belle-famille active l'obligation de différer le contre-don, de manière à maintenir l'illusion de la gratuité comme le rappelle autant La Rochefoucauld (« Le trop grand empressement que l'on a de s'acquitter d'une obligation est une espèce d'ingratitude ») que Bourdieu $» .^{11}$

11 La différence proposée ici entre soirée amicale et soirée familiale repose sur une application du principe d'obligation : en autorisant les contre-dons immédiats, les hôtes amicaux libèrent leurs invités de l'inscription de leurs relations dans la longue durée, celle de l'irréversibilité des rapports familiaux. Mais ce raccourcissement du délai entre don et contre-don n'implique pas la dénonciation d'un intérêt caché ou d'une intention larvée d'obliger: on est simplement quitte, on ne se doit rien. Par contre, au sein du système familial, les membres n'en ont jamais fini de se devoir toujours quelque chose. Leurs échanges s'étalent, s'organisent et prennent sens dans le temps - le temps d'une vie. On peut se demander, parvenu à ce stade du raisonnement, si les opérations de relations publiques ne fonctionnent pas plutôt sur le mode familial des dons et contredons, en raison du fait qu'elles placent les invités dans l'impossibilité de rendre rapidement ce qu'ils ont reçu, si bien qu'ils se retrouvent pour longtemps en position d'obligés de leurs hôtes.

12 On le voit : nous sommes ici en pleine spéculation - fragile, mal assurée encore, irritante. Mais c'est bien là son rôle : donner à penser. Cette stratégie de rupture n'est jamais aussi 
efficace que lorsqu'elle fonctionne de concert avec son homologue inversé : la démarche microsociologique.

13 L'observation (participante ou non) des comportements qui se produisent au sein de manifestations de relations publiques peut constituer, par l'objectivation des rapports qu'elle offre, une quatrième stratégie de rupture. Il est facile de rapporter un événement de relations publiques aux «rites d'interaction» d'Erving Goffman. C'est d'ailleurs un «remplisseur conversationnel» de choix lorsque les "acteurs" présents aiment se donner l'illusion qu'ils font partie des cognoscenti ${ }^{12}$. Il est beaucoup moins aisé d'opérationnaliser les notions plutôt que les concepts de Goffman ${ }^{13}$, du moins lorsqu'on se donne comme objectif d'aller au-delà des oppositions simples inspirées de l'analyse dramaturgique (région antérieure et postérieure, façade et coulisse, etc.). Ainsi, la très riche notion d'« engagement " (involvement) que Goffman élabore à plusieurs reprises dans son œuvre $\aleph^{14}$, permet à l'observateur attentif de guider son regard et de repérer à tout instant d'une interaction des éléments comportementaux pertinents. En effet, une des difficultés de l'observation ethnographique de lieux publics ou semi-publics est que l'on voit à la fois beaucoup et pas assez. Il ne se passe en général « rien d'extraordinaire », comme dit la langue commune. Pour lire la banalité, c'est-à-dire pour voir des microévénements à tout instant, il faut un regard informé théoriquement, un regard qui pose des questions et cherche des réponses. À partir de ce moment-là, tout devient simple - et très complexe. Simple, parce que l'on « sait » regarder ; complexe, parce que les données affluent subitement en trop grand nombre. Ainsi, dès le moment où l'on se pose la question de l'engagement des participants à une manifestation de relations publiques dans leur interaction, on peut, par exemple, chercher à savoir :

14 - Comment les signes de cet engagement sont répartis sur différentes régions du visage et du corps (yeux plissés, hochements de la tête, épaules tendues, etc.) ;

15 - Comment s'établissent, dans le jeu interactionnel, les réponses mutuelles à l'engagement ( «je fais attention à ce que vous dites et je vous le montre en gardant mon regard dans le vôtre »);

16 - Comment les interruptions dans l'engagement peuvent apparaître (e.g. détournements répétés du regard), amenant l'interaction de l'« euphorie » à la « dysphorie »; - Comment les " pare-engagements » s'élaborent à partir des objets présents (colonnes, palmiers en pot, etc ;) ou des personnes présentes :

18 - Etc

19 Etudier les modes et les signes d'engagement dans une manifestation de relations publiques peut répondre à deux objectifs très différents: soit on tente de cerner les comportements d'engagement d'une manière générale, les manifestations de relations publiques n'étant qu'un lieu commode de collecte de données; soit on cherche à déterminer la spécificité de l'engagement lors de cette "occasion sociale " particulière qu'est la manifestation de relations publiques (ce sont les aspects « situationnels » du comportement, dit Goffman). Mais quels que soient les objectifs que l'on se fixe, l'observation ethnographique, sinon éthologique, de manifestations de relations publiques a pour résultat induit de les faire apparaître sous un jour non tant désenchanté qu'immobile : les acteurs peuvent changer, leurs gestes sont tous identiques, d'un lieu à l'autre, d'un jour à l'autre. Alors que les manifestations de relations publiques reflètent, selon les bons manuels, la «culture d'entreprise» de l'hôte, la micro-analyse laisse 
apercevoir une sorte d'idiome institutionnel commun: mêmes paroles de bienvenue, mêmes sourires, mêmes poignées de main.

Bien sûr, les relations publiques ne se réduisent pas à leurs manifestations. La métaphore de l'iceberg devrait à nouveau être rappelée ici. Mais il reste que les relations publiques produisent des activités publiques à la fois visibles, accessibles et immédiatement avouables (contrairement à certaines formes de lobbying). Les appréhender à travers leurs manifestations est donc de bonne guerre.

Les stratégies proposées ici sont donc tantôt très empiriques (approches historique et ethnographique), tantôt très spéculatives (rapprochements historiques et " iconoclastes »); elles visent tantôt les discours que produisent les relations publiques sur elles-mêmes, tantôt les pratiques qu'elles engendrent. Cet éclatement importe peu; l'essentiel est de maintenir le cap sur un objectif : montrer qu'en matière de relations publiques, rien ne peut être taken for granted, accepté comme allant de soi, dès lors qu'on veut travailler scientifiquement.

Ce principe s'illustre encore dans la cinquième stratégie, qui remonte à la source même de l'idée de relations publiques: la communication entre les différentes parties concernées par l'entreprise ou l'institution initiatrice. L'ensemble des discours et des pratiques de relations publiques repose sur un modèle de la communication plus ou moins explicite, celui de la transmission ponctuelle de messages verbaux entre un «émetteur » et un «récepteur ». Ce modèle est si prégnant qu'il est devenu la réalité même des relations publiques : elles ne se pensent qu'à partir de l'image du réseau de flèches circulant en tous sens. Que se passerait-il si l'on changeait de modèle? Or cette possibilité existe ${ }^{15}$. Le modèle dit "orchestral » élaboré par des anthropologues comme Bateson, Birdwhistell ou Hall (cf La Nouvelle Communication, op. cit.) permet de penser la communication autrement. Appliqué aux relations publiques, il permet de les envisager de manière non plus instrumentale mais relationnelle. Les professionnels deviennent ainsi des " opérateurs symboliques ", chargés de l'âme de l'entreprise " ${ }^{16}$, de son histoire et de sa dynamique. Bien sûr, c'est du rêve éveillé; mais le contraste avec la réalité permet de mieux saisir les limites de pensée et d'action que se sont donné sans nécessité les relations publiques.

\section{De la rupture à la construction de l'objet}

«S'imposer une polémique incessante contre les évidences aveuglantes qui procurent à trop bon compte l'illusion du savoir immédiat et sa richesse indépassable ", comme disaient encore Bourdieu, Chamboredon et Passeron ${ }^{17}$, n'est jamais qu'une première étape dans la mise en place d'une problématique proprement scientifique. Dans le cas qui nous occupe, construire l'objet « relations publiques " passe par des mises à distance : il faut oser s'éloigner des relations publiques pour éventuellement mieux y revenir. Éventuellement, car rien ne dit que la réflexion, théoriquement, historiquement et ethnographiquement fondée, ne va pas amener le chercheur à dessiner un autre espace, c'est-à-dire à construire un objet qui ne cerne plus les contours des relations publiques telles que ses acteurs les voient. À la vérité, il serait étonnant que l'objet construit ressemble encore dans ses limites conceptuelles à celui dont il a procédé empiriquement. Rien ne dit non plus que la reconfiguration des relations publiques se fera sur un seul modèle. On peut envisager une insertion de l'étude des relations publiques dans celle, plus générale, des relations en public; on peut aussi les concevoir dans le cadre d'une 
"théorie générale de l'économie des pratiques" et non d'une "théorie des pratiques proprement économiques»(P. Bourdieu); une anthropologie des rites de passage pourrait encore les accueillir, tout comme une histoire contemporaine des modes de représentation. L'objectif serait de les insérer dans une problématique plus vaste qui leur offrirait un statut d'objet scientifique.

Des théories de relations publiques : pour quoi faire? La question mérite d'être posée in fine car, en sachant retourner l'iconoclasme comme soi, il faut se demander si quelqu'un écoute. Dans les milieux professionnels, on a certainement d'autres chats à fouetter : dans les milieux scientifiques et universitaires, les relations publiques ne constituent certainement pas un objet d'études prioritaire... Il faut donc non seulement construire l'objet, mais le public. D'où viendrait-il ? Sans doute d'abord de cette nouvelle génération de jeunes universitaires aujourd'hui dans les relations publiques. Ils sont mieux formés que leurs aînés, plus ouverts à la réflexion. Ils n'interpréteront pas d'emblée une démarche de "rupture » comme une attaque contre la profession. Ils ne chercheront pas à dénigrer les universitaires qui n'ont pas leur expérience du terrain. Ils pourront accepter un discours sans "application" - inutile, en quelque sorte. Il reste à espérer que, du côté scientifique, un public se constitue également, ouvert à l'idée que les relations publiques sont une des métaphores sociales essentielles de la fin de ce siècle.

\section{NOTES}

1. P. Bourdieu. J.-C. Chamboredon, J.-C. Passeron. éds. Le Métier de sociologue. Paris, Mouton/ Bordas, 1968 (nouvelle édition. 1973 ; actuellement distribué par W. De Gruyter à Berlin). Quatre sociologue proches de Bourdieu ont récemment réécrit Le Métier de Sociologue en s'appuyant sur leurs propres travaux : P. Champagne, R. Lenoir, D. Merllié. L. Pinto, Initiation à la pratique sociologique, Paris, Dunod, 1989. Pierre Bourdieu, qui reconnaît lui-même qu'il a écrit son ouvrage "pour faire le point sur un état de [sa] recherche " (Magazine Littéraire, $\mathrm{N}^{\circ} 303$, octobre 1992, p. 63) a reformulé sa vision du «métier de sociologue» dans «La pratique de l'anthropologie réflexive (le séminaire de Paris)», in P. Bourdieu et L.J.D. Wacquant, Réponses, Parts. Éd. du Seuil, 1992, pp. 187-231.

2. Du moins nombre d'entre eux, une acerbe critique du Métier de Sociologue est développée par R. Miguelez, « La rupture en sociologie », L'Homme. Oct.-Déc 1970, pp. 111-123

3. Il faut rappeler combien la pensée de Georges Canguilhem est importante dans la genèse de celle de Bourdieu - et celle de très nombreux intellectuels français des années soixante. Michel Foucault l'a superbement dit dans un texte peu connu: «cet homme dont l'œuvre austère, volontairement bien délimitée, et soigneusement vouée à un domaine particulier dans une histoire des sciences qui, de toutes façons, ne passe pas pour une discipline de grand spectacle, s'est trouvé d'une certaine manière présent dans les débats où lui même a bien pris garde de ne jamais figurer. Mais ôtez Canguilhem et vous ne comprenez plus grand chose à toute une série de discussions qui ont eu lieu chez les marxiste français : vous ne saisissez pas, non plus, ce qu'il y a de spécifique chez des sociologues comme Bourdieu. Castel. Passeron, et qui les marque si fortement dans le champ de la sociologie ; vous manquez tout un aspect du travail théorique fait chez les psychanalystes et en particulier chez les lacaniens. Plus : dans tout le débat d'Idée qui a 
précédé ou suivi le mouvement de 68 , il est facile de retrouver la place de ceux qui de près ou de soin, avaient été formés par Canguilhem» (in «La vie: l'expérience et la science ». Revue de Métaphysique et de Morale, $\mathrm{n}^{\circ}$ 9, 1985. pp. 3-4

4. Je me suis livré à cet exercice dans d'autres textes, notamment dans «L'insaisissable statut théorique des relations publiques» (in G. Ringlet éd, Guide des médias. Deurne, Kluwer, 1990, Suppl. 6 pp. 1-16) et «Communication et relations publiques: quelles théories pour quelles pratiques? » (à paraître in B. Mangelinckx, éd. Communication et relations publiques. Bruxelles. De Boeck).

5. J'ai ainsi récemment eu le plaisir de diriger à Liège un mémoire de fin d'études de second cycle sur l'émergence d'un discours professionnel « RP » en Belgique francophone dans les années 50 : Sandrine Jacquet, Catholicisme social et relations publiques : éléments pour une histoire des relations publiques en Belgique dans les années 50 et 60 , Université de Liège, mémoire en Arts et Sciences de la Communication, dactyl, 1992. L'étude n'est pas définitive, mais elle permet déjà de bien comprendre les rapports idéologiques entre le monde social-chrétien belge et les relations publiques du moins au niveau des discours fondateurs. Il resterait notamment à définir dans cette optique le rôle joué par l'Université Catholique de Louvain, première université belge francophone à offrir un enseignement des relations publiques.

6. Ainsi l'ouvrage du sociologue Luc Boltanski, Les Cadres : la formation d'un groupe social (Paris, Éd. de Minuit. 1982) a fourni la trame de l'étude de S. Jacquet, op. cit.

7. Je ne veux pas entrer ici dans un débat extrêmement complexe qui convoquerait autant Aron que Foucault, Veyne que Hayden White (The Content of the Form : narrative Discourse and Historical Représentation Baltimore, The John Hopkins University Press, 1987). Si la «micro-histoire » réduit singulièrement la distance entre auteurs et professionnels de l'histoire (Éd. Muir et G Ruggiero, eds, Microhistory and the Lost Peoples of Europe, Baltimore, The John Hopkins University Press, 1991, qui présente l'«école» de Quaderni Storici: Carlo Cinzburg et l'équipe du «Séminaire de Bologne »), à l'instar de l'anthropologie narravive américaine dea années 80 (cf. I. Brady, ed. Anthropological Poetics, Savage, Maryland. Rowman and Littlefield. 1991), il reste d'une part que ces travaux sont menés par des chercheurs professionnels, qui introduisent nécessairement une « institution du savoir » (comme disait Michel de Certeau dans "L'opération historique », in J. Le Goff et P. Nora (Sous la dir. de), Faire de l'histoire, Paris, Gallimard, 1974, pp. 3-41) et, d'autre part, que ces rapprochements ne sont pas encore produits en histoire contemporaine et encore moins en histoire contemporaine de la vie économique, sociale et culturelle, à laquelle pourrait ressortir une histoire des relations publiques. La distance peut être minimale : peu importe - la rupture est consommée des lors qu'il y a dédoublement.

8. Axel Gryspeerdt. "Vous êtes des nôtre » : tentatives d'élargissement de la sphère interne de l'entreprise par la communication et les comportements de relations publiques, à paraître dans la revue Comunicaçao e linguagem (Lisbonne).

9. Y. Winkin. "L'insaisissable statut théorique des relations publiques", art. cit. p. 111 par "professionnels de la représentation», l'entendais «non seulement les publics relations, mais encore les journalistes, les hommes politiques, les mandataires publics, qui consacrent une partie importante de leur temps a être physiquement présents en divers lieux publics et à faire savoir qu'ils y sont ».

10. M. De Coster. L'analogie en sciences humaines, Paris, Presses Universitaires de France, 1978, p. 26.

11. «...le fonctionnement de l'échange de dons suppose la méconnaissance de la vérité du «mécanisme» objectif de l'échange, celle-là même que la restitution immédiate dévoile brutalement: l'intervalle de temps qui sépare le don et le contre-don est ce qui permet de percevoir comme irréversible une structure d'échange toujours menacée d'apparaître et de s'apparaître comme réversible, c'est-à-dire comme à la fois obligée et intéressée » (P. Bourdieu, Esquisse d'une théorie de la pratique, Genève, Librairie Droz. 1972. p. 223). 
12. «Aussi longtemps qu'il n'a pas rendu, celui qui a reçu est un obligé, tenu de manifester sa gratitude envers son bienfaiteur ou, en tout cas, d'avoir des égards pour lui, de le ménager (...)» (P. Bourdieu, op. cit, p. 223-24).

13. Je ne fais allusion ici qu'au Goffman de Mise en Scène de la vie quotidienne (Paris, Éd. de Minuit, 1973) et de Rites d'interaction (Paris, Éd. de Minuit. 1974) : le Goffman des Cadres de l'expérience (Paris, Éd. de Minuit, 1991) est certainement plus fécond encore, mais sa maîtrise est très ardue au point que personne, ou presque, ne l'exploite systématiquement en Europe comme aux ÉtatsUnis.

14. Je me permets ici de renvoyer le lecteur aux pages que j'ai consacrées à cette notion dans Erving Goffman : les Moments et leurs Hommes (Paris, Éd. du Seuil et Éd. de Minuit, 1988, pp. 75-77) ainsi qu'au chapitre 3 ( «Involvement, Interdependence and Alienation») de l'ouvrage de Tom Burna, Erving Goffman (London, Rontlgedge, 1992, pp. 48-75). Le texte « Engagement », repris dans la Nouvelle Communication (Paris. Éd. du Seuil, 1981, pp. 267-278), offre une première bonne approche de la notion: "Être impliqué dans une activité de circonstance signifie y tenir une certaine attention intellectuelle et affective, une certaine mobilisation de ses ressources psychologiques; en un mot, cela signifie s'y engager » (p. 270).

15. J'ai développé cette argumentation dans un autre texte ( Communication et relations publiques : quelles théories pour quelles pratiques? » art cit ), je me permettrai donc d'être très bref ici.

16. Pour faire une allusion non voilée au livre d'Alain Etchegoyen, Les entreprises ont-elles une âme ?, Paris Éditions François Bourin. 1990.

17. P. Bourdieu. J.-C. Chamboredon et J.-C. Passeron, op. cit. p. 27.

\section{RÉSUMÉS}

Les relations publiques restent encore de l'ordre du savoir-faire («savoir faire savoir », comme dit la formule consacrée), leur enseignement relève ainsi le plus souvent de la transmission de principes, préceptes et présupposés issus de l'expérience pratique de professionnels. Du point de vue de la recherche en communication, une double opération doit être menée afin de transformer cet objet social en un objet scientifique : rompre avec les discours ambiants et oser proposer des premières élaborations pleinement théoriques. Plusieurs stratégies de rupture sont proposées et rapportées à des travaux de recherche, qui montrent que les relations publiques peuvent effectivement changer de statut épistémologique.

Public relations are still in the domain of practical know-how (to know how to make known, as the French formula says). Their teaching has most often to do with the transmission of principles, recipes and presuppositions drawn from the everyday experience of public relation officers. From the perspective of research in communication, a double edged operation must be launched in order to transform that social object into a scientific object: on the one hand, one must break with the discourses floating around; on the other hand, one must offer fully theorical elaborations. Several strategies for break-up are offered and meshed with actual research projects, which show that public relations can effectively overhaul their epistemologica! status. 


\section{AUTEUR}

\section{YVES WINKIN}

Yves Winkin est « chargé de cours » au Département des sciences philosophiques et des sciences de la communication de l'Université de Liège. Il dirige le laboratoire d'anthropologie de la communication, qui se spécialise dans l'approche ethnographique des rites d'interaction en milieu urbain. Il a publié en 1981 une anthologie raisonnée aux Editions du Seuil, intitulée La nouvelle Communication (Collection Points $\left.n^{\circ} 136\right)$. Ses travaux plus récents ont notamment porté sur la trajectoire intellectuelle et sociale d'Erving Goffman (Les moments et leurs hommes. Paris. Seuil/Minuit. 1988). Il a dirigé la section « Communication interpersonnelle et interculturelle » du Dictionnaire Critique de la Communication (Paris. Presses Universitaires de France. 1993 ; Lucien Sfez. directeur). 